\title{
Influence of boundary layer state on development downstream of normal shock interactions
}

\author{
Todd S. C. Davidson* \& Holger Babinsky ${ }^{\dagger}$ \\ Engineering Department, University of Cambridge, Trumpington Street, Cambridge, CB2 1PZ, UK
}

\begin{abstract}
Flat plate experiments investigating flow development downstream of normal shock wave-laminar boundary layer interactions are reported. Laminarity is desirable for low drag, but problems are expected at shock interactions due to separation, leading to unsteadiness and large boundary layer growth. A flat plate with elliptical leading edge profile is used to generate a laminar boundary layer, which encounters a Mach 1.2 normal shock at a Reynolds number of $1.6 \times 10^{6}$. Upstream of the interaction, the boundary layer is too thin for detailed measurement, but schlieren images are presented and laser Doppler velocimetry results reported downstream. For comparison, similar measurements are then made with trips located upstream. Far downstream, shape factor and skin friction are seen to be independent of incoming boundary layer state, contrary to expectations: although separation does occur in the laminar case, it is very thin and the anticipated impact on the boundary layer health is not seen. In fact, an equilibrium turbulent boundary layer is recovered far sooner downstream and is thinner than in the tripped cases, which exhibit attached turbulent interactions. In summary, normal shock wave-laminar boundary layer interactions did not exhibit the anticipated detrimental effects, and instead appear quite benign.
\end{abstract}

\section{Nomenclature}

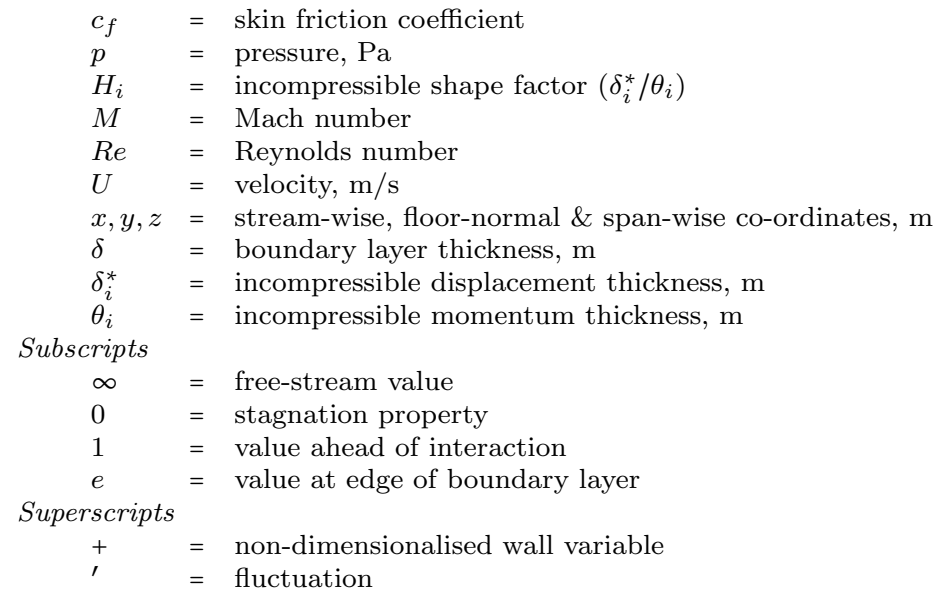

\footnotetext{
*Former Research Student, Aero Lab; Member AIAA - correspondence: todd.davidson@cantab.net

${ }^{\dagger}$ Professor of Aerodynamics; Associate Fellow AIAA

Presented as Paper 2016-0044 at the 54th AIAA Aerospace Sciences Meeting, San Diego, CA, 4-8 January 2016. Copyright (C) 2017 Todd S. C. Davidson and Holger Babinsky. Published by the American Institute of Aeronautics and Astronautics, Inc., with permission.
} 


\section{Introduction}

There are a number of locations on high speed aircraft where boundary layers encounter shock waves, influencing both internal and external aerodynamics. One particular example is on transonic wings, where the supersonic region on the upper surface is terminated by a normal shock wave which interacts with the boundary layer. Due to increasing economic and environmental challenges, interest in laminar boundary layers is on the rise - as a drag-reducing design choice on wings; and as a consequence of higher altitudes and lower Reynolds numbers in internal flows.

Laminar boundary layers have the advantage of lower skin friction, but separate easily in the presence of adverse pressure gradients. Separation can lead to significant increases in drag and reduced functionality of control surfaces in external flows, and increased total pressure losses and turbulence in internal flows. It is an inherently unsteady phenomenon and can cause large scale fluctuations in the flow, such as transonic buffet on aerofoils, which can limit flight envelopes. It is clear then that separation is generally to be avoided, but there is a dearth of prior research into normal shock interactions with laminar or transitional boundary layers, and in particular how shock-induced laminar separation can affect the flow development downstream of an interaction.

Classically, researchers of shock wave-boundary layer interactions (SBLIs) have focused on turbulent cases, $[1,2]$ with interactions generally categorised as 'attached' or 'separated'. For normal shock strengths of up to Mach 1.3 or 1.4, a turbulent boundary layer typically stays attached through the interaction. The shock presents a large adverse pressure gradient to the flow, which penetrates through the boundary layer. As this pressure rise reaches the subsonic region of the boundary layer, it is communicated upstream. This means that the shock is 'felt' upstream and the boundary layer begins to thicken. This thickening changes the direction of the outer supersonic flow, causing compression waves which transmit the increasing pressure. Where these waves meet the main shock, near the surface, they reduce its strength as more of the compression has already occurred, and deflect it rearwards to stay normal to the turned flow. This process is visible in schlieren imaging as the incident shock wave 'smearing' into a shock foot near the wall, with a length of the order of the boundary layer thickness (Fig. 1a). [1-7]

For stronger shocks, the pressure rise transmitted upstream is so great that the boundary layer cannot negotiate it without separating. A Mach number of at least 1.3 is typically quoted for turbulent separation, corresponding to a pressure rise of more than $80 \%$. [3] A separation bubble forms, presenting a relatively sharp change in direction which propagates through the outer supersonic flow as an oblique shock. The bottom of the incident shock is then angled downstream of the intersection to again remain approximately 

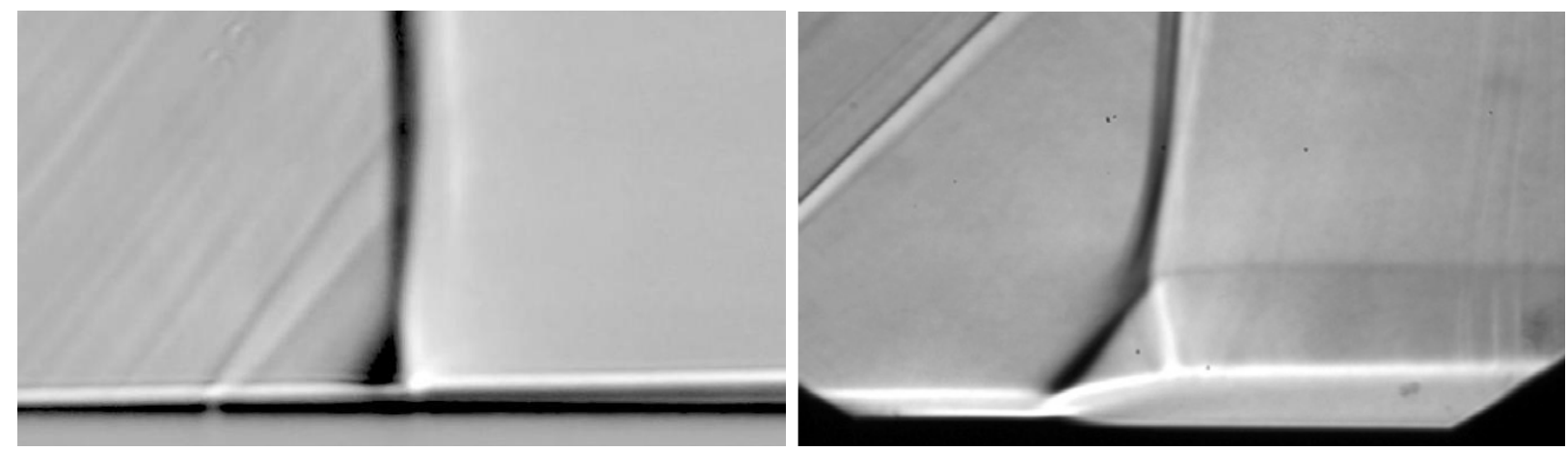

Fig. 1 Schlieren images of normal shock wave-turbulent boundary layer interactions: a) attached, $\left.M_{\infty}=1.28, R e_{x_{\text {shock }}}=1.79 \times 10^{6} ;[8] \mathrm{b}\right)$ separated, $M_{\infty}=1.5, R e_{x_{\text {shock }}}=2.15 \times 10^{6}$. [9]

normal to the local flow, forming a characteristic $\lambda$-shock with the outer normal shock and the oblique 'separation shock' (Fig. 1b). The length of the lambda structure is of the order of a few boundary layer thicknesses. $[2-7,10-12]$

There have been few experimental studies of interactions between laminar boundary layers and normal shock waves. However, the theory of Messiter, Feo \& Melnik (1971) [13] predicts that the pressure jump required to separate laminar boundary layers at transonic speeds is $R e_{x}{ }^{-0.2}$ - less than $10 \%$ for $R e_{x}>1 \times 10^{5}$, equivalent to a normal shock Mach number of only 1.05. Above this shock strength, a lambda structure appears, with the front leg transmitting this separation pressure. For increasing Mach number, the separation length and size of the shock structure are predicted to increase, though with no change in the front leg pressure jump. The authors' analysis further suggests that this laminar separation pressure will be spread across a sufficient streamwise distance such that the front leg of a laminar interaction lambda shock structure will be a collection of compression waves rather than having coalesced into an oblique shock as in separated turbulent interactions. [13]

The canonical normal laminar SBLI experiment was conducted by Ackeret, Feldman \& Rott [1] in 1947 using a variable geometry transonic wind tunnel, with a supersonic region forming over a convex plate, terminated by a quasi-normal shock. Figures $2 \& 3$ reproduce the laminar interaction at Mach 1.23 \& $R e_{x_{\text {shock }}}=0.43 \times 10^{6}$, showing a lambda shock and separation - superficially similar to a separated turbulent interaction, but at a much lower shock strength. The pressure rise through the front leg was $7.5 \%$, matching the value predicted by Messiter et al. [13] Further interactions with shock strengths from 1.1 to 1.3 were shown, each with separation and a lambda shock, and a similar pressure rise through the front leg. The general conclusions of various investigators are that the shock structures of laminar interactions are larger than their turbulent counterparts, as more of the boundary layer is subsonic; separation is far more likely; and secondary shocks are more common. $[1,11,14,15]$ 
As shown in Figs. $2 \&$ 3, a secondary shock was present behind the main shock in Ackeret et al., although the authors could not determine whether this was inherent to the laminar SBLI or an artefact of the floor curvature. Transition was identified ahead of the rear shock at $x=195 \mathrm{~mm}$, apparently by an increase in fluctuations within the boundary layer. However, it was reported that blockage of the boundary layer probe strongly influenced the flow and so, in general, further measurements were unreliable.

Laminar separation investigations of a very different nature, such as the DNS study by Alam \& Sandham (2000) of an incompressible boundary layer in the presence of a strong adverse pressure gradient, have also observed the pattern of a small pressure rise through the separation, then transition occurring over the separation bubble, followed by the bulk of the surface pressure increase, with reattachment downstream. [16]
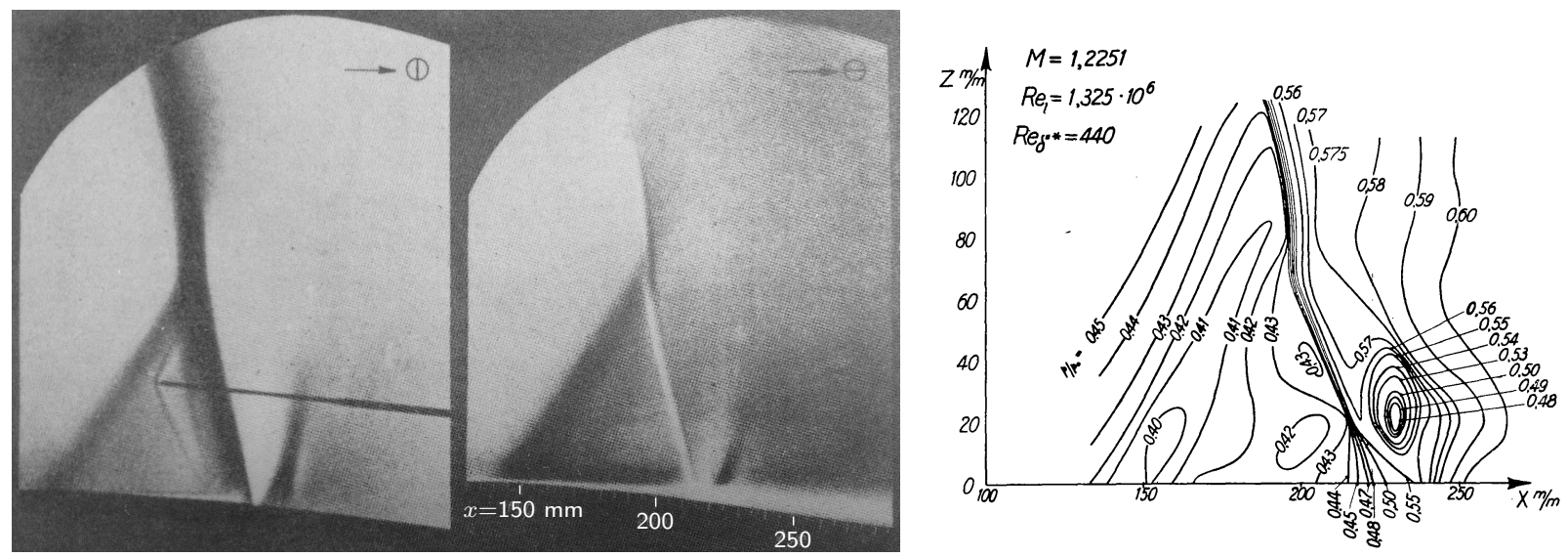

Fig. 2 Ackeret et al.'s schlieren images of a normal Fig. $3 \quad p / p_{0}$ isobars through the inlaminar SBLI, $M_{\text {shock }}=1.23, R e_{x_{\text {shock }}}=0.43 \times 10^{6}$. [1] teraction of Fig. 2. [1]

Little information was reported downstream of Ackeret et al.'s interaction and so few conclusions can be drawn about the overall effect on the flow field, leaving a number of questions unanswered on normal shock-laminar/transitional boundary layer interactions. In this paper, the problem is revisited using modern experimental techniques, to both further our knowledge and aid future computational work (in which transitional flows and separation remain challenging). This research aims to examine the impact of the interaction on the boundary layer downstream, which may have important practical implications and was not investigated by Ackeret et al. Key questions are how damaging is the laminar separation to the flow downstream, in terms of unsteadiness and boundary layer health; and by how much can the flow be improved by tripping the boundary layer to avoid separation. These will be judged by laser Doppler velocimetry (LDV) measurements of the incompressible integral parameters downstream of laminar and tripped interactions. As such, an experiment has been developed to hold a normal shock wave on a flat surface (to isolate the interaction from the curvature of Ackeret et al.'s geometry) at a Mach number of 1.2 - sufficient to separate a laminar boundary layer, but too weak for turbulent separation. The interaction Reynolds number is $1.6 \times 10^{6}$, representing the lower range of transonic aerofoils [17] and the upper range of compressor blades [18]. 


\section{Experimental facility and techniques}

Experiments were conducted in the CUED No. 1 Supersonic Tunnel, an intermittent blow-down facility with run times up to 30 seconds and a working section $179 \mathrm{~mm}$ high \& $114 \mathrm{~mm}$ wide. The stagnation pressure is manually controlled during runs, with $<0.1 \%$ fluctuation. Hot-wire anemometry was performed using a $5 \mu \mathrm{m}$ diameter Dantec 55P11 probe and StreamLine Pro anemometer to quantify the level of free steam turbulence. The probe was mounted in the free steam of the otherwise empty tunnel with subsonic liners, and operated at high overheat ratios so as to be sensitive only to mass-flux fluctuations, from which velocity fluctuations are estimated using Smits' model. $[19,20]$ For transonic tests, the Mach number is controlled by varying the downstream blockage (Fig. 5) and the probe's response calibrated by varying the stagnation pressure. [21, 22] Figure 4 shows the intensities and spectra for three Mach numbers. [8] The $-5 / 3$ decay can be clearly seen, and there seems to be a trend of decreasing turbulence with increasing Mach numbers, though further investigation would be needed to confirm this. The general magnitudes agree well with past results $(<1 \%$ at Mach $0.8[23]$ and $0.58 \%$ at Mach $1.3[24])$.

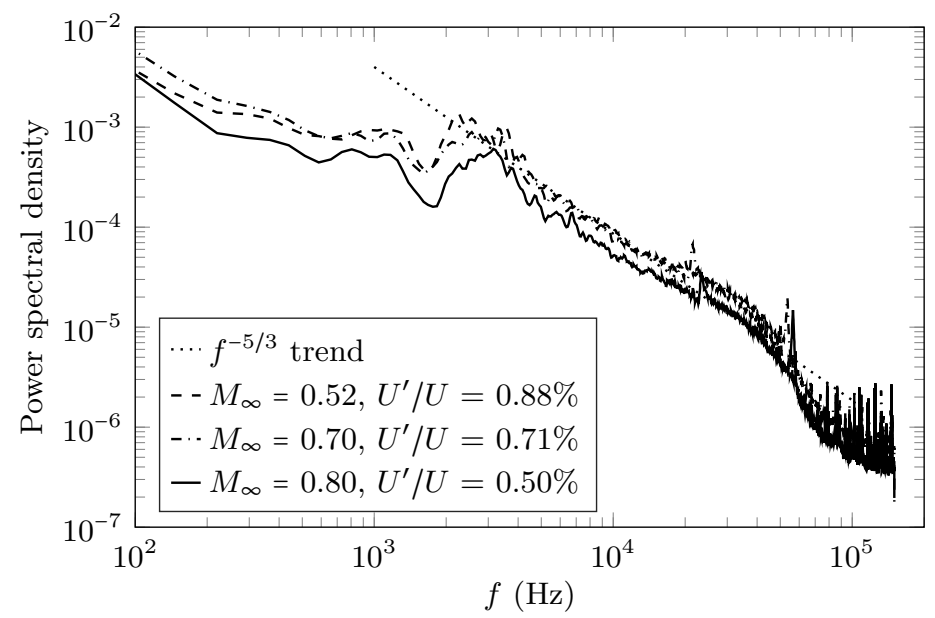

Fig. 4 Turbulence spectra showing decreasing intensities with increasing Mach number.

The experimental configuration is sketched in Fig. 5, with a flat plate held in a transonic free stream. The $12 \mathrm{~mm}$ thick plate has a 6:1 elliptical profile around the leading edge (Fig. 6a), shaped by wire-erosion, and was hand-polished to a surface roughness of $R_{a}<0.2 \mu \mathrm{m}$. The free steam is accelerated by the curvature of the ellipse and a supersonic region develops around the leading edge of plate, terminated by a shock. The profile ensures a favourable pressure gradient throughout the initial boundary layer development to maintain laminarity. The plate has a slight angle of attack $\left(\sim+0.2^{\circ}\right.$, measured with a digital inclinometer $)$ to counteract the blockage effect of boundary layer growth along the tunnel side-walls and the floor below is cut away to avoid choking the flow. The shock position can be accurately controlled during each run by an adjustable blockage element downstream. 


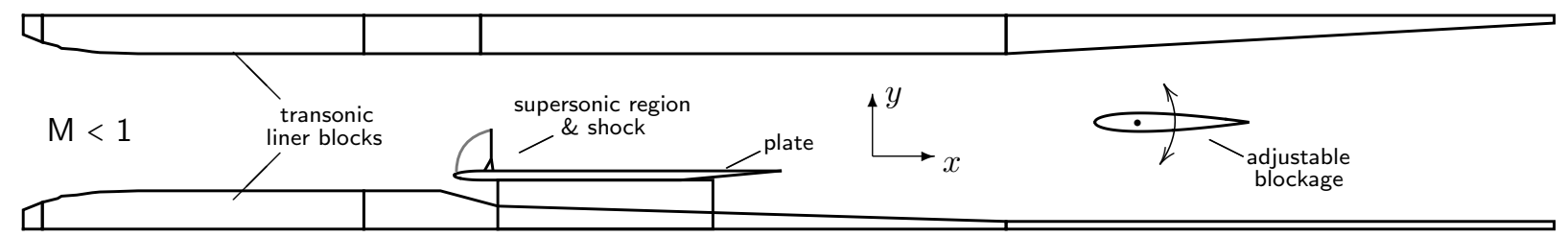

Fig. 5 Schematic of the tunnel layout. The orientation of the co-ordinate system is shown, with $x=0$ at the leading edge, $y=0$ at the plate's flat surface.
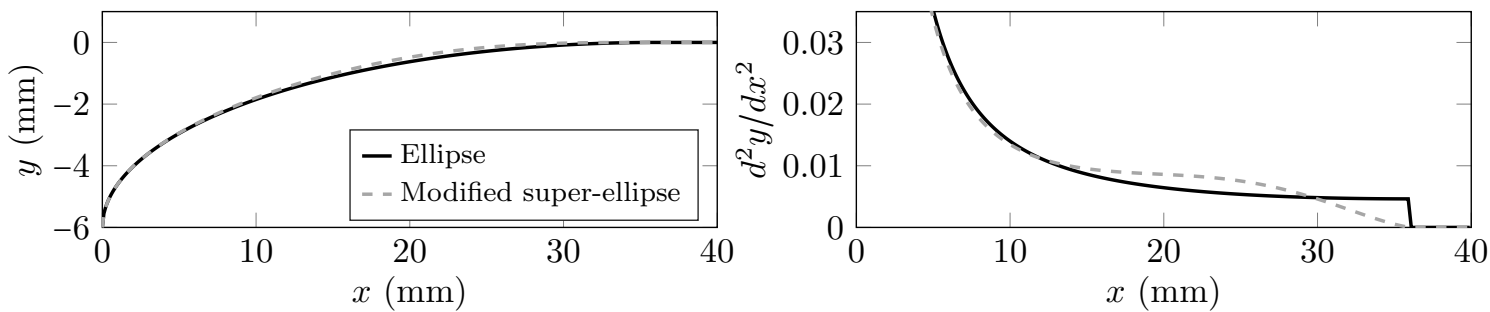

Fig. 6 a) Leading edge profiles for the standard ellipse and modified super-ellipse profiles with an aspect ratio of 6 . b) Surface curvature for the two profiles. [25]

A second flat plate was manufactured with a modified super-ellipse (MSE) profile around the leading edge.

This shape, sketched in Fig. 6a, avoids the discontinuity in curvature at the end of the elliptical section (Fig. 6b), and other researchers have reported that such a profile can delay transition. [e.g. 25, 26]

Two styles of tripping device were applied at $x=20 \mathrm{~mm}$ on the elliptical leading edge flat plate and measured with a Taylor-Hobson 'Form TalySurf' contact profilometer, which also confirmed leading edge profiles and determined surface roughness. The first is a two-dimensional step, created by a strip of gloss spray paint across the plate, $2 \mathrm{~mm}$ wide and $6 \mu \mathrm{m}$ high. This was the smallest trip that could be repeatably produced. For context, a $0.1 \mathrm{~mm}$ thick Blasius profile has $\delta^{*}{ }_{i}=28 \mu \mathrm{m}$ and $\theta_{i}=11 \mu \mathrm{m}$, and so the evidence of Fig. 13 suggests the trip height is of a similar order to the momentum thickness at the same location.

The second tripping method is three-dimensional, with drops of PVA glue carefully applied at the centre-span $(z=0)$ and at $10 \mathrm{~mm}$ intervals across the plate. Uniformity of the dots could not be achieved, with heights of 90-180 $\mathrm{\mu m}$ (mean $120 \mu \mathrm{m}$ ), and diameters of 1.4-2.2 mm (mean $1.7 \mathrm{~mm}$ ). The spacing was determined according to van Driest \& Blumer (1961) [27] and Bernardini et al. (2014) [28].

\section{A. Flow measurement}

A 'Z-type' schlieren system is used to visualise the flow [29], with images captured at 6,000 fps by a Photron FASTCAM ultima APX high speed camera fitted with a $180 \mathrm{~mm}$ Nikon lens, and a horizontal knife-edge. Imperfections in the optical path are removed by subtracting a 'wind-off' background image from each 'windon' picture. Variation in intensity across a line of pixels for each frame is used to extract shock position versus time, and the fluctuation throughout a run is analysed to detect any shock unsteadiness. 
A two-component laser Doppler velocimetry (LDV) system from TSI is used in forward-scatter mode to measure velocities. The emitter and receiver are mounted on either side of the tunnel on the pedestals of a three axis traverse, which uses stepper motors with a resolution of $0.01 \mathrm{~mm}$ for the $x \& z$ directions and 0.005 $\mathrm{mm}$ in the floor-normal $y$ direction. A pinhole jig on the tunnel centre-line is used to confirm the crossing position within the tunnel. The traverse is positioned so that the measurement volume intersects the pinhole, which is evidenced by the lasers refracting through the small gap, creating an interference pattern of fringes. For further accuracy of position, the floor location is checked before each run. The beam intersection is moved towards the surface until a clear reflection becomes visible in the receiving optics viewfinder. By positioning this bright patch so it is equally strong on both sides of a target, the local floor height is found.

The emitter is mounted so the measured vectors are at $45^{\circ}$ to $x \& y$ and the lower beam pair is angled slightly below horizontal. The $363 \mathrm{~mm}$ lens was chosen for its small measurement volume, producing beam overlap waists of $90 \& 85 \mu \mathrm{m}$ for the green and blue channels respectively. The corresponding fringe spacings are $3.7 \& 3.6 \mu \mathrm{m}$, and the span-wise beam overlap lengths are $1.3 \& 1.2 \mathrm{~mm}$. The receiving head points slightly down and downstream to reduce observed reflections. Results are recorded by TSI's FlowSizer software using a band-pass filter of $40-120 \mathrm{MHz}$ to capture velocities from 0 to $300 \& 284 \mathrm{~m} / \mathrm{s}$.

Droplets of paraffin, $\sim 0.5 \mu \mathrm{m}$ in diameter (as determined by a shock-lag method), are injected through a rake in the settling chamber and provide good seeding levels around the centre-span, following the flow well. [30] In the free stream, valid burst rates are in the range of $25-40 \mathrm{kHz}$, although this drops dramatically in the boundary layer. Averages are taken over $0.2 \mathrm{~s}$ at each measurement position, which typically ensures at least 5,000 samples in the bulk flow. However, very near the wall (below $0.1 \mathrm{~mm}$ ) this can fall as low as 500 per data point. Samples outside of three standard deviations are rejected for the purposes of determining the mean and r.m.s. velocities. Across the span, only the central $20 \%$ is adequately seeded.

The manufacturer-quoted accuracy on particle velocity is $0.1 \%$ of the band-pass filter's maximum, or $0.5 \%$ of averaged velocities. [31] Further errors are introduced by the emitting head pitch of $-3.3^{\circ}$ and rotation of $45^{\circ}$, which have an accuracy of $\pm 1 \%$ each which affects the measured velocities. Span-wise velocities, $w$, are generally expected to be an order of magnitude smaller than $v$ and are neglected throughout. The errors that this generates are greatest in separated regions, where all velocity components can be of similar size, but as there is little seeding in these regions, reliable measurements cannot be taken anyway; and downstream of the trip dots, where vortices can induce span-wise velocities of a similar magnitude to $v$.

Another notable source of error in the LDV technique is velocity biasing. Put simply, higher velocities carry more particles through the measurement volume, and a point measurement of a fluctuating velocity 
will show a higher apparent average velocity as more droplets will be detected during positive fluctuations. McLaughlin et al. (1973) [32] found this error to be $10 \%$ of the local fluctuation intensity, and so below $0.2 \%$ of the velocity in much of the flow, increasing through the boundary layer up to $4 \%$ near the wall.

This bias is exacerbated by velocity gradients across the measurement volume: within a boundary layer, higher velocities near the top will register more seeding counts than low velocities at the bottom, and so the mean velocity supposedly measured at each point will be greater than the true value. This is compounded by the reduction in seeding levels lower in the boundary layer. In summary of all of these effects; the typical total accuracy of measured velocities is taken to be $\pm 2 \%$; except in the areas with strong span-wise flows mentioned above, where it approaches $\pm 5 \%$; and within $0.1 \mathrm{~mm}$ of the floor, where low seeding, reflections and the measurement volume intersecting the floor increase the error level to $10 \%$.

\section{B. Fitting turbulent boundary layer profiles}

The LDV system is primarily used to measure boundary layers, which can be characterised by the incompressible integral parameters: displacement thickness, $\delta_{i}^{*}$; momentum thickness, $\theta_{i}$; and shape factor, $H_{i}$. The incompressible quantities are used as they more faithfully quantify the kinematic behaviour of the boundary layers and as such are far more prevalent in the literature for low supersonic flows, simplifying comparisons of profiles at different Mach numbers. [3]

Integrating directly from LDV data to calculate these quantities suffers from two drawbacks: the first is the gaps between data points, which would be integrated by assuming straight lines between data and so represent a less full profile; and the greater is that the system is unable to measure very close to the wall, as seen in Fig. 7. This is due to a number of factors: the lack of seeding particles close to the surface; the bright reflections that occur when near the plate; and the measurement volume itself intersecting the floor at the bottom of the boundary layer.
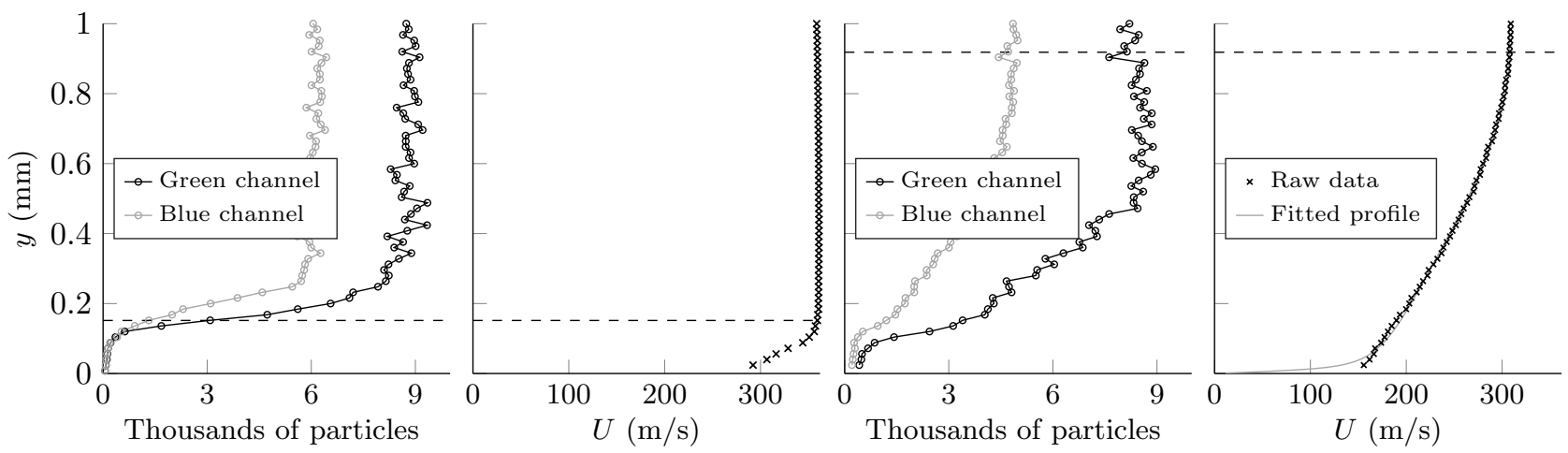

Fig. 7 Boundary layer seeding rates and velocity data ( $\delta$ at black dashes):

a\&b) upstream of the clean interaction, $x-x_{\text {shock }}=-12 \mathrm{~mm}$;

c\&d) downstream of the $2 \mathrm{D}$ trip interaction, $x-x_{\text {shock }}=+5 \mathrm{~mm}$. 


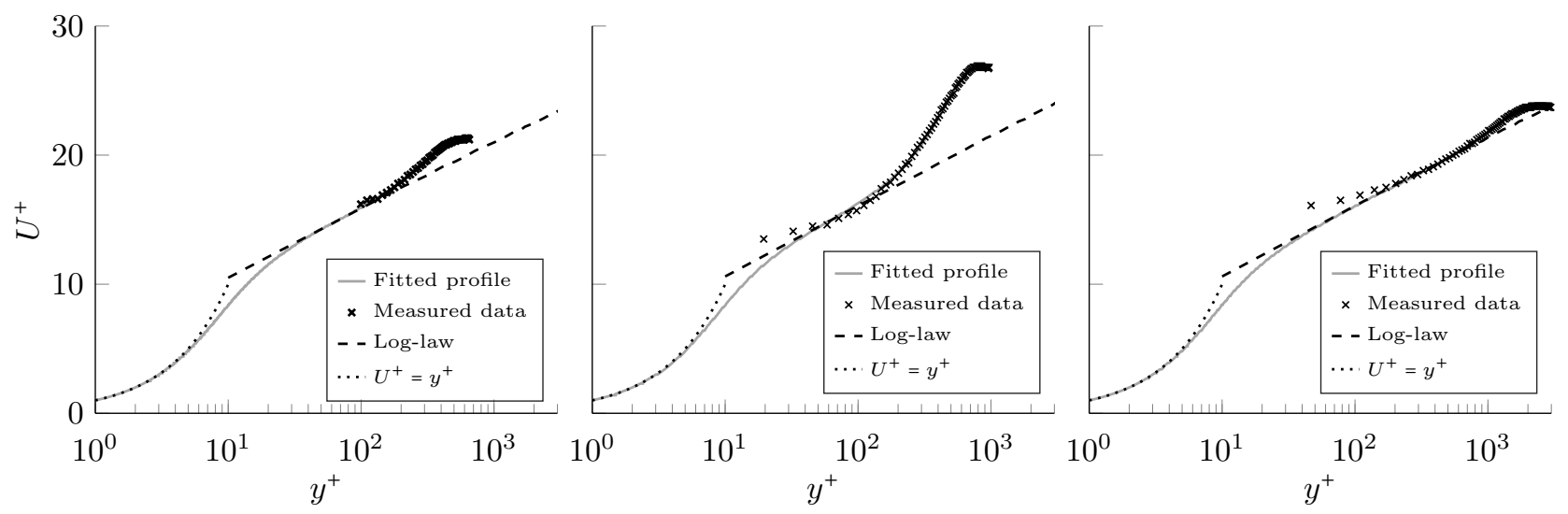

Fig. 8 Examples of fitted turbulent profiles in logarithmic wall units:

a) downstream of a 3D trip dot, before the interaction, $x-x_{\text {trip }}=25 \mathrm{~mm}, x-x_{\text {shock }}=-3$ $\mathrm{mm}, z=0: \delta=0.57 \mathrm{~mm}, c_{f}=3.47 \times 10^{-3}, R_{\text {fit }}^{2}=0.999$;

b) shortly downstream of the interaction with $2 \mathrm{D}$ trip, $x-x_{\text {shock }}=+5 \mathrm{~mm}$, also seen in Fig. $7 \mathrm{~d}: \delta=0.92 \mathrm{~mm}, c_{f}=2.35 \times 10^{-3}, R^{2}$ fit $=0.998$;

c) far downstream of the clean interaction, $x-x_{\text {shock }}=+102 \mathrm{~mm}$, also seen in Fig. 14b: $\delta=$ $2.27 \mathrm{~mm}, c_{f}=2.97 \times 10^{-3}, R^{2}$ fit $=0.999$.

Figure 7a illustrates the difficulties of measuring thin, laminar boundary layers. Only eight points have been achieved within the boundary layer, and the data is questionable for all the reasons discussed above. As a result of this, only the thickness is retained upstream of the laminar interactions, with no further attempt to discern the profile or integral properties. More generally, a cut-off of $\delta>0.5 \mathrm{~mm}$ is imposed for the use of fitted profiles and calculated integral properties (discussed below) for all boundary layer measurements.

In order to improve the calculation of the integral parameters, previous researchers have fitted analytical profiles to the measured data to more faithfully represent turbulent boundary layers. The technique implemented in this paper relies on the Sun \& Childs (1973) [33] method, based on the classical combination of the logarithmic law-of-the-wall [34] and the Coles wake function [35]. The model is matched to experimental data using MATLAB's built-in fitting procedures to vary $\delta, c_{f}$ and $U_{e}$ to line the profile up with measurement points, which are weighted by their seeding counts. The viscous sub-layer $\left(U^{+}=y^{+}\right.$, up to $\left.y^{+}=5\right)$ and buffer zone (up to $y^{+} \sim 100$ ) are then incorporated into the profile with the method of Musker (1979) [36], using $c_{f}$ from the Sun \& Childs fit. A number of examples of fitted profiles generated by the combination of these two models are plotted in logarithmic co-ordinates in Fig. 8 , from a variety of situations. Figure 8b shows a boundary layer just downstream of a tripped interaction, with the wake function extending almost all the way down to the buffer region, and the skin friction coefficient is low; whereas Fig. 8a\&c show equilibrium turbulent profiles. The model provides an excellent fit, with $R^{2}$ coefficients greater than 0.99 , even in regions of strong adverse pressure gradients. The incompressible integral parameters calculated from the fitted profiles are deemed to be accurate to within $5 \%$ of the true values. [37] 


\section{Flat plate interactions}

\section{A. Schlieren images}

A schlieren image of the interaction on the clean standard ellipse flat plate is shown in Fig. 9a. White expansion waves can be seen around the elliptical profile as the flow accelerates to supersonic speeds, which end at the start of the flat section of the plate $(x=36 \mathrm{~mm}$, marked by a vertical white line on the image). The characteristic angle of the waves suggests a Mach number around 1.2. Four millimetres downstream of end of curvature there is a smeared dark wave (from $x=40 \mathrm{~mm}$ at the plate) which forms the front leg of a lambda shock structure (whose legs meet around $y=18 \mathrm{~mm}$ ), implying the presence of a separation bubble, just as in Ackeret et al. The rear leg of the shock structure is more distinct than the front, and points slightly downstream from the intersection. The free-stream shock position is $x=51 \mathrm{~mm}$.

Separation can be inferred from the presence of the lambda structure, which, combined with the low shock strength, implies that the boundary layer is laminar. The schlieren image also indicates that the boundary layer ahead of the shock is less than $1 \mathrm{~mm}$ thick, whereas the interaction has a streamwise length of around $17 \mathrm{~mm}$ and so is several times larger, providing further confirmation that the boundary layer is not turbulent, as even separated turbulent interaction lengths are rarely more than $\mathcal{O}(5 \delta)$. [5, 38] There is no secondary shock present, contrary to the observations of Ackeret et al., which suggests that it was induced by surface curvature in that experiment and is not an inherent feature of normal laminar SBLIs.

The shock structure is steady: high speed schlieren imaging shows a standard deviation of position of less than $0.5 \mathrm{~mm}$. The streamwise shock location can be controlled by varying the downstream blockage (Fig. 5). If driven further upstream, the shock enters the acceleration region and so its strength reduces, until only a series of shocklets remain. If the shock is moved downstream to $x=70 \mathrm{~mm}$, as seen in Fig. 10, the lambda structure is replaced by only the smeared shock foot of an attached turbulent interaction, suggesting that the boundary layer naturally transitions shortly downstream of the shock location of Fig. 9a.

A schlieren image with the modified super-ellipse plate installed is shown in Fig. 9b, with a steady interaction again. The apparent intensity of the expansion waves is significantly reduced towards the end of the profile, as there is less curvature in this region of the modified super-ellipse shape, which helps to visualise the front leg of the lambda shock. Otherwise, the interaction looks much the same as on the standard ellipse plate, with a streamwise length of around $16 \mathrm{~mm}$. Unfortunately, the characteristic lambda structure of a laminar interaction was not observed any further downstream with this plate as was hoped, which may be due to the comparatively high Reynolds number. (Despite its similarity to the standard ellipse flat plate interaction, 

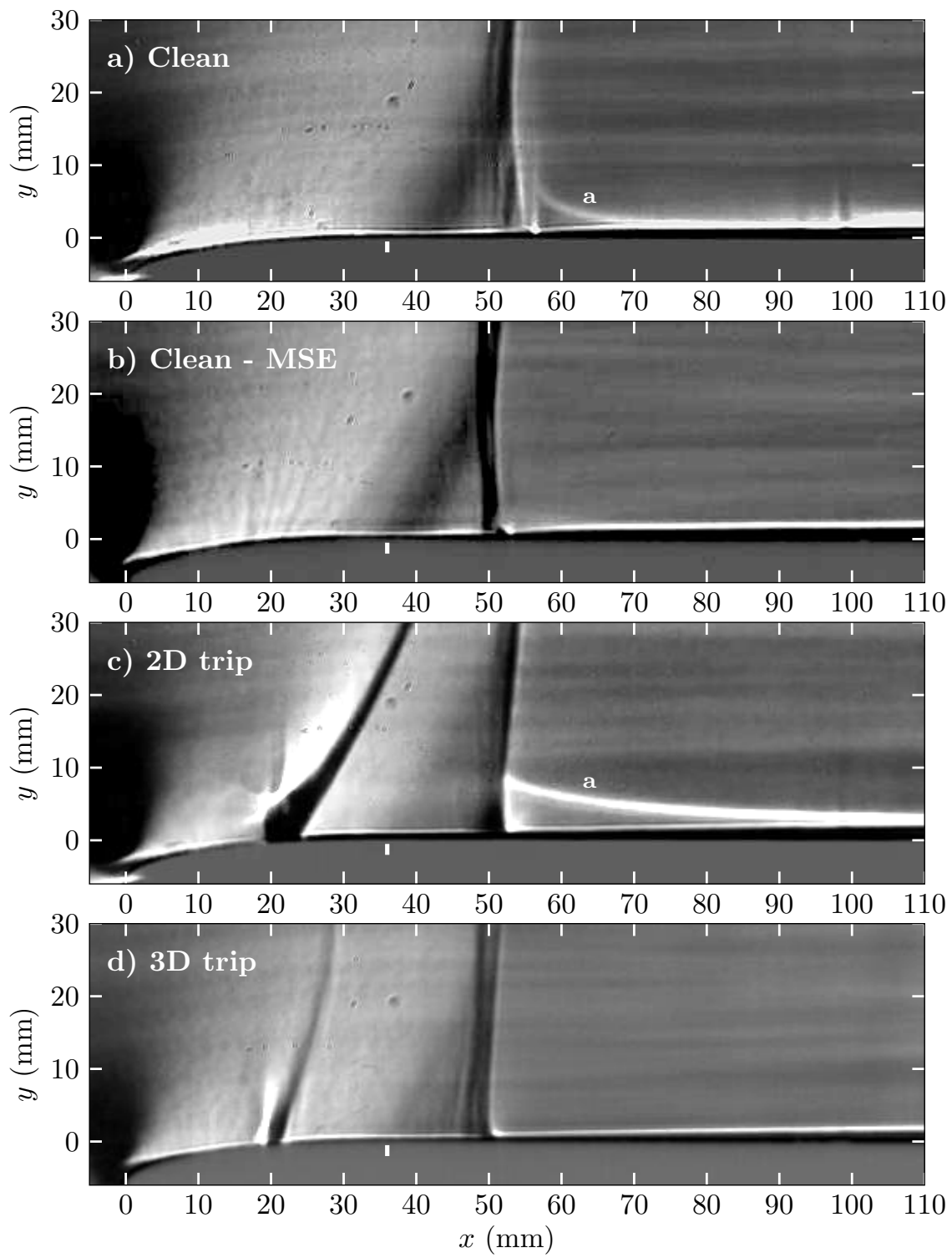

Fig. 9 Schlieren images (white lines mark the end of curvature at $x=36 \mathrm{~mm}$ ):

a) clean interaction on the standard ellipse flat plate, $R e_{x_{\text {shock }}}=1.60 \times 10^{6} ;^{a}$

b) clean interaction on the MSE flat plate, $R e_{x_{\text {shock }}}=1.57 \times 10^{6}$;

c) standard ellipse flat plate with $2 \mathrm{D}$ trip at $x=20 \mathrm{~mm}, R e_{x_{\text {shock }}}=1.63 \times 10^{6}$; $^{\text {a }}$

d) standard ellipse flat plate with $3 \mathrm{D}$ trip at $x=20 \mathrm{~mm}, R e_{x_{\text {shock }}}=1.57 \times 10^{6}$.

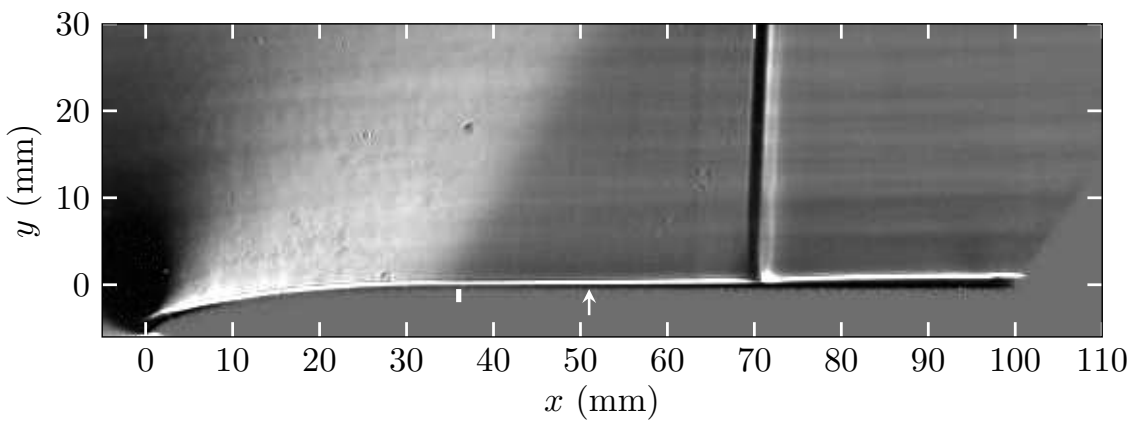

Fig. 10 The clean standard ellipse plate with blockage reduced, moving the shock downstream, $R e_{x_{\text {shock }}}=2.20 \times 10^{6}$ (white arrow indicates shock position in Fig. 9a).

\footnotetext{
${ }^{a}$ The white line curving towards the plate present in some schlieren images is an optical phenomenon resulting from the exact alignment of the collimated beam, the highly polished flat plate surface and the boundary layer density variation. [8, 39-42] It was removed in later experiments by a mechanical iris positioned around the focus of the light path.
} 
the data set is still included as it was acquired using a set of tunnel walls with increased optical access, which permitted the measurement of profiles further downstream, as seen later in Fig. 13.)

Figure $9 \mathrm{c}$ shows the resulting flow when a strip of paint is added at $x=20 \mathrm{~mm}$ to trigger transition on the standard ellipse plate, with an intense wave generated over the trip. At the normal shock, no lambda structure is formed, and instead a typical steady attached turbulent interaction is observed, with a streamwise width of the shock foot of approximately $5 \mathrm{~mm}$ (as estimated from the schlieren image by comparison with the thickness of the plate). The strength of the normal shock interaction was unaffected by the trip, as indicated by the angle of the expansion waves at the end of the curvature and later confirmed by LDV measurements, despite the apparent strength in the schlieren image of the generated shock.

A schlieren image is given in Fig. 9d of the flow with trip dots installed at $x=20 \mathrm{~mm}$ on the standard ellipse plate. Bow shocks are expected to form around each dot, but these are not resolved in Fig. 9d as the schlieren technique integrates across the span. The wave seen at the trip location is less intense than in Fig. 9c, due to the reduced span-wise extent and the integration effect; but steeper, due to the greater height of the trips causing higher local flow deflection. The normal shock is again steady and has little smearing at the interaction, with a shock foot only $4 \mathrm{~mm}$ long.

\section{B. Laser Doppler velocimetry investigation}

LDV is employed to gather more information about the strength of the interactions. Figure 11 presents a streamwise traverse at $1 \mathrm{~mm}$ above the clean standard ellipse plate flat surface, which gives the peak Mach number as 1.18. The Mach number then drops to 1.14 through the front leg and from 1.11 down to 0.93 through the rear shock, giving nominal static pressure increases of $5.3 \% \& 23.7 \%$ respectively (neglecting
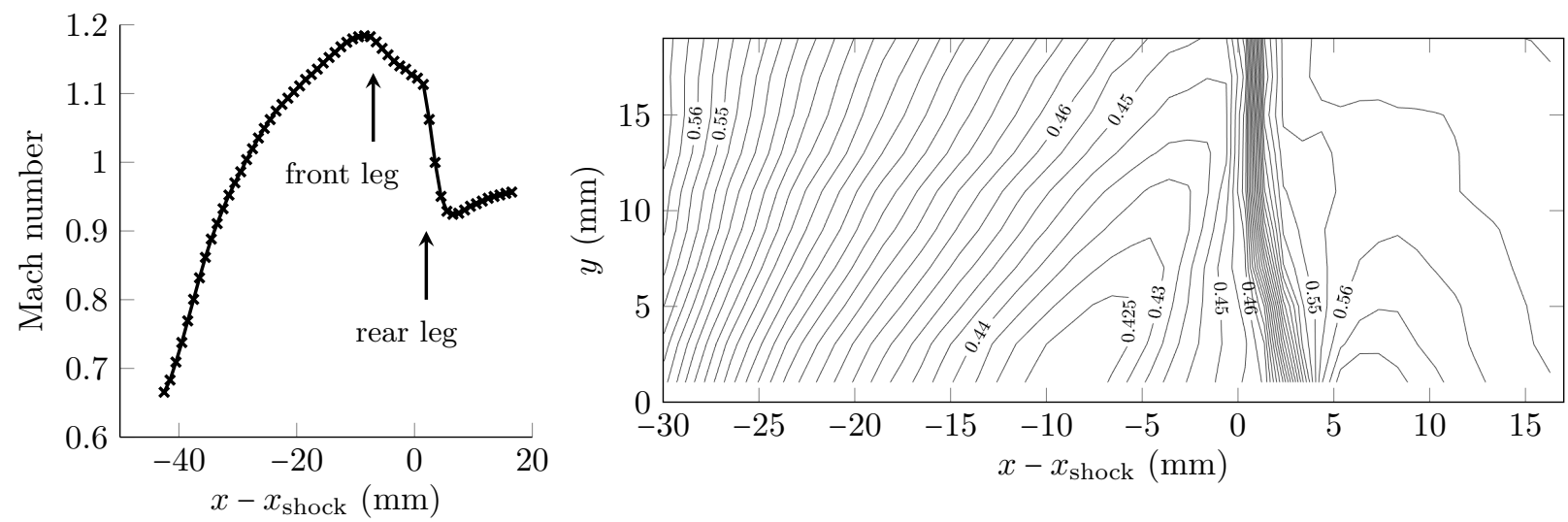

Fig. 11 LDV traverse at $y=1$ Fig. $12 p / p_{0}$ isobars ( 0.005 between contours $)$ from LDV $\mathrm{mm}$ through the clean interaction. through the interaction of Fig. 9a (c.f. Fig. 3). 
Table 1 Flat plate interaction details ( $\delta_{1}$ is measured just upstream of the interaction, and so has a high degree of uncertainty in the 'Clean' and 'MSE' cases).

\begin{tabular}{c|cccccc} 
Interaction & $M_{\text {peak }}$ & $p_{0}(\mathrm{bar})$ & $\mathrm{Unit} R e(/ \mathrm{m})$ & $R e_{x_{\text {shock }}}$ & $R e_{\delta_{1}}$ & $\delta_{1}(\mathrm{~mm})$ \\
\hline Clean & 1.18 & 2.05 & $31.4 \times 10^{6}$ & $1.60 \times 10^{6}$ & 6,300 & 0.2 \\
Clean - MSE & 1.20 & 2.05 & $31.5 \times 10^{6}$ & $1.57 \times 10^{6}$ & 3,200 & 0.1 \\
2D trip & 1.19 & 2.05 & $31.4 \times 10^{6}$ & $1.63 \times 10^{6}$ & 22,000 & 0.7 \\
3D trip & 1.18 & 2.05 & $31.4 \times 10^{6}$ & $1.57 \times 10^{6}$ & 18,800 & 0.6
\end{tabular}

stagnation pressure losses, which would be only $0.5 \%$ for a Mach 1.18 normal shock). The prediction by Messiter et al. (1971) of a $R e_{x}{ }^{-0.2}$ pressure increase for shock-induced laminar separation equates to $5.9 \%$ for the present interaction, in good agreement with the observed rise. [13] Further measurements are built into a pressure contour map in Fig. 12, similar to that of Ackeret et al. in Fig. 3. Table 1 presents the details of each of the interactions of Fig. 9.

The LDV system has been used to investigate the boundary layer development along the centre-line of the plate for each flow case, which can be summarised by comparing the distributions of boundary layer thickness, shape factor and skin friction coefficient in Fig. 13 (recalling that where $\delta$ is less than $0.5 \mathrm{~mm}$, only the boundary layer edge location is retained and no attempt is made to determine profiles - see Fig. 7). For the 3D trip case, measurements are presented at $z=-5 \mathrm{~mm}$ (in between two dots) in addition to $z=0$ (in line with the central dot), due to the inherent spanwise variation.

Ahead of the shock, the tripped boundary layers are much thicker than the clean baseline. Downstream of the interaction, the 2D trip case still shows the greatest boundary layer thickness, followed by the measurements made behind a trip dot. The laminar interactions show a downstream boundary layer thickness thinner than or equal to the tripped cases, despite undergoing separation, the lambda shock and reattachment.

Figure 13b shows the development of the shape factors along the plate. For the clean cases, the LDV technique was unable to measure far enough upstream to determine the transition positions or detect the large $H_{i}$ expected in separated flow. However, downstream of the laminar interactions the shape factor is between 1.3 and 1.4 at all locations, indicating reattached healthy turbulent boundary layers with no detrimental impact of the shock.

The tripped cases show a spike in $H_{i}$ shortly after the incident shock position (although the peaks are well below the value of 2.6 typically associated with turbulent separation [2]) and then exhibit a long recovery back to equilibrium turbulent boundary layer behaviour, as expected for attached turbulent interactions, e.g. Délery (1985) [2], eventually converging with the clean case data. The skin friction coefficients generated in the Sun \& Childs fitting procedure are also plotted in Fig. 13c, where the measurements taken between trip dots reveal an intermediary behaviour between the clean and turbulent interactions. 

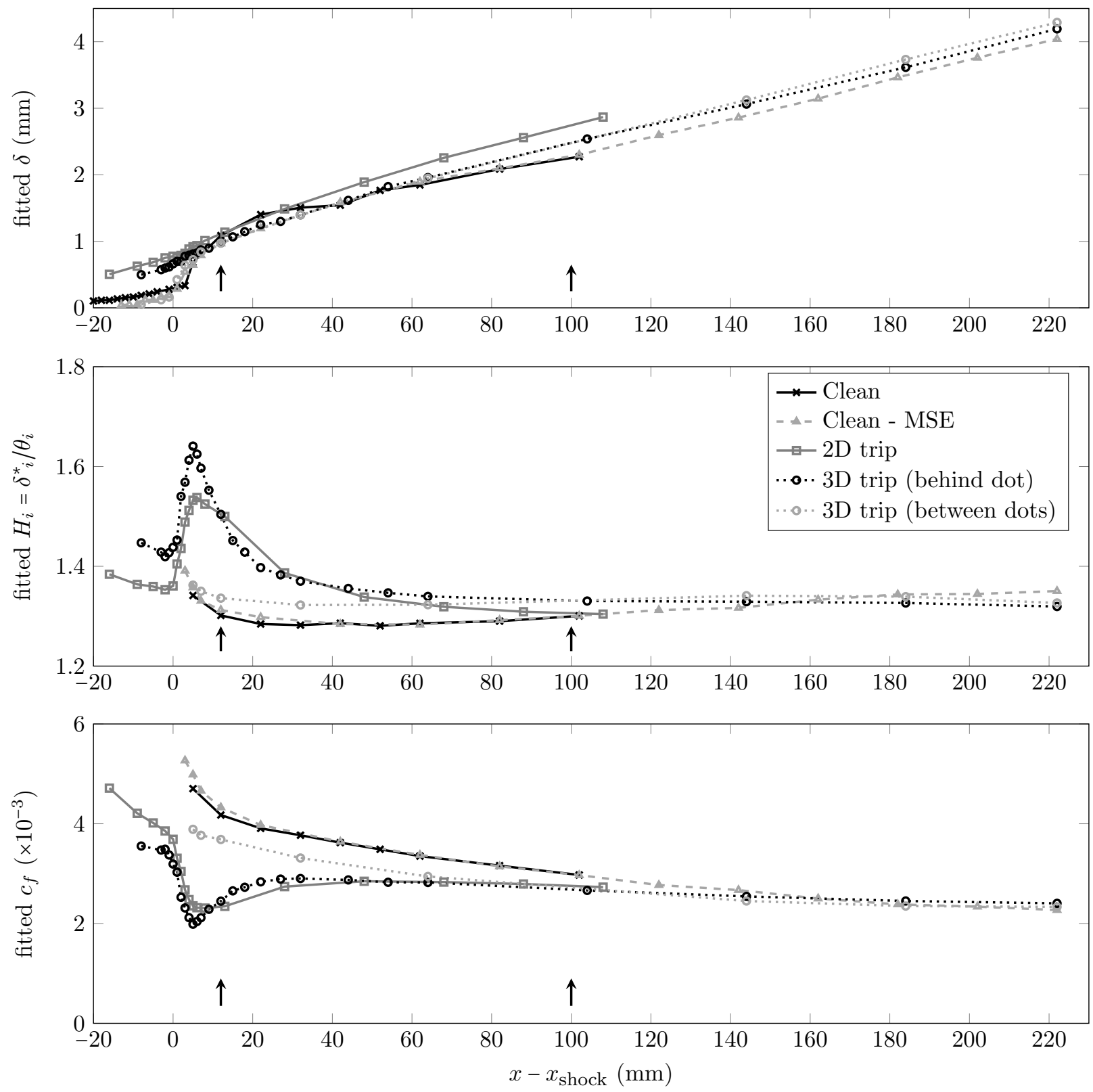

Fig. 13 Fitted turbulent boundary layer properties for the flat plate interactions: a) boundary layer thickness; b) incompressible shape factor; c) skin friction coefficient. Arrows mark the locations of the profiles in Figs. $14 \& 15$.

Two locations are selected to illustrate the differences in downstream development between the clean and tripped interactions. Figure 14a presents normalised profiles from around $12 \mathrm{~mm}$ behind the normal shock position. The two clean cases show almost identical profiles of a full, equilibrium turbulent boundary layer, corresponding to the shape factor of 1.3 seen in Fig. 13b. In contrast, the equivalent measurements taken behind the trips show profiles that are much less full, with large wake function contributions (see Fig. 8b). The profile taken between two trip dots again shows an intermediate distribution. Further downstream (Fig. 14b, 100 mm behind the shock), all five profiles have converged to a typical turbulent equilibrium shape. 

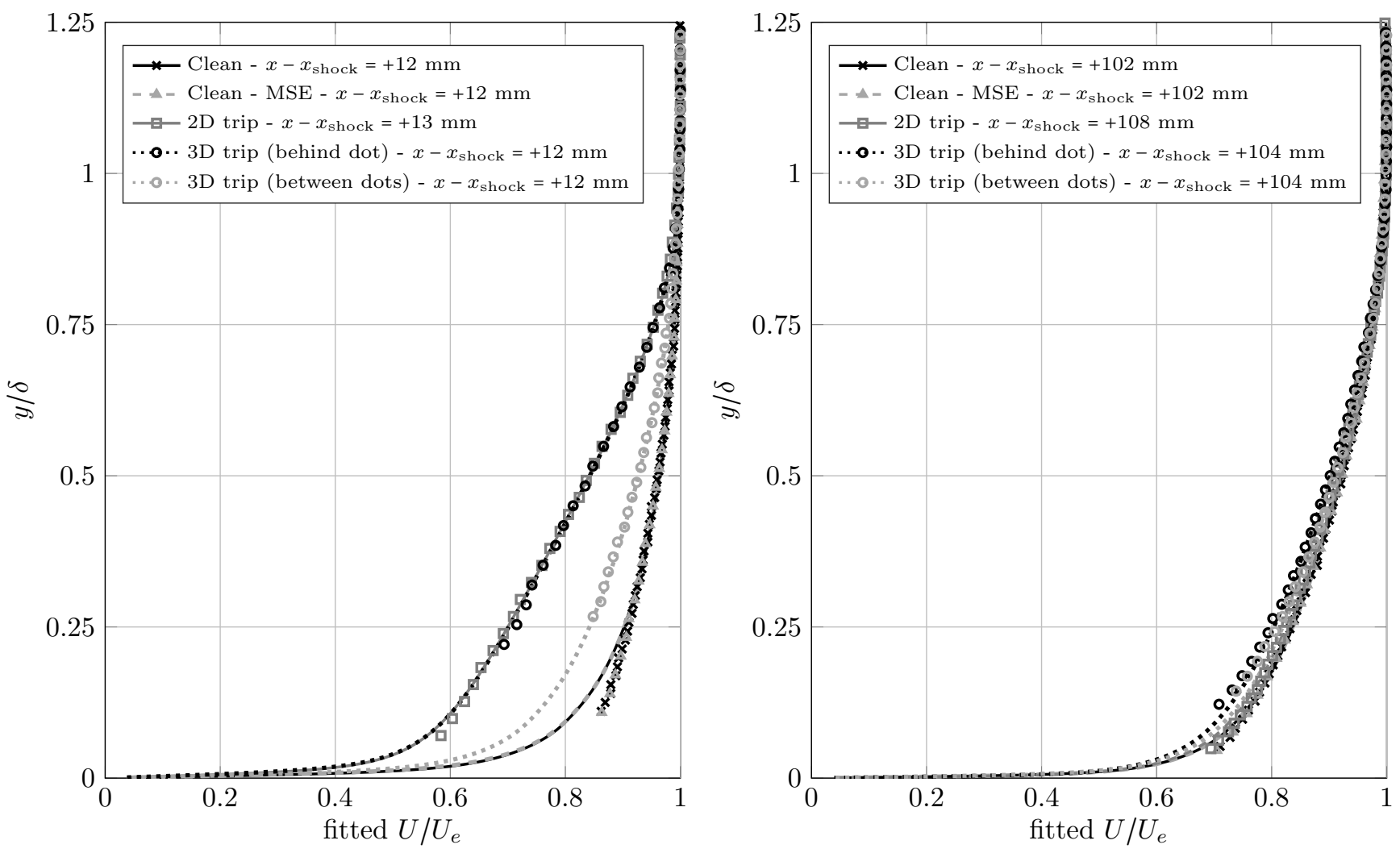

Fig. 14 Raw measurements (marks) and their fitted turbulent boundary layer profiles (lines) downstream of the interaction for each case: a) $x-x_{\text {shock }} \sim+12 \mathrm{~mm}$; b) $x-x_{\text {shock }} \sim+100 \mathrm{~mm}$.
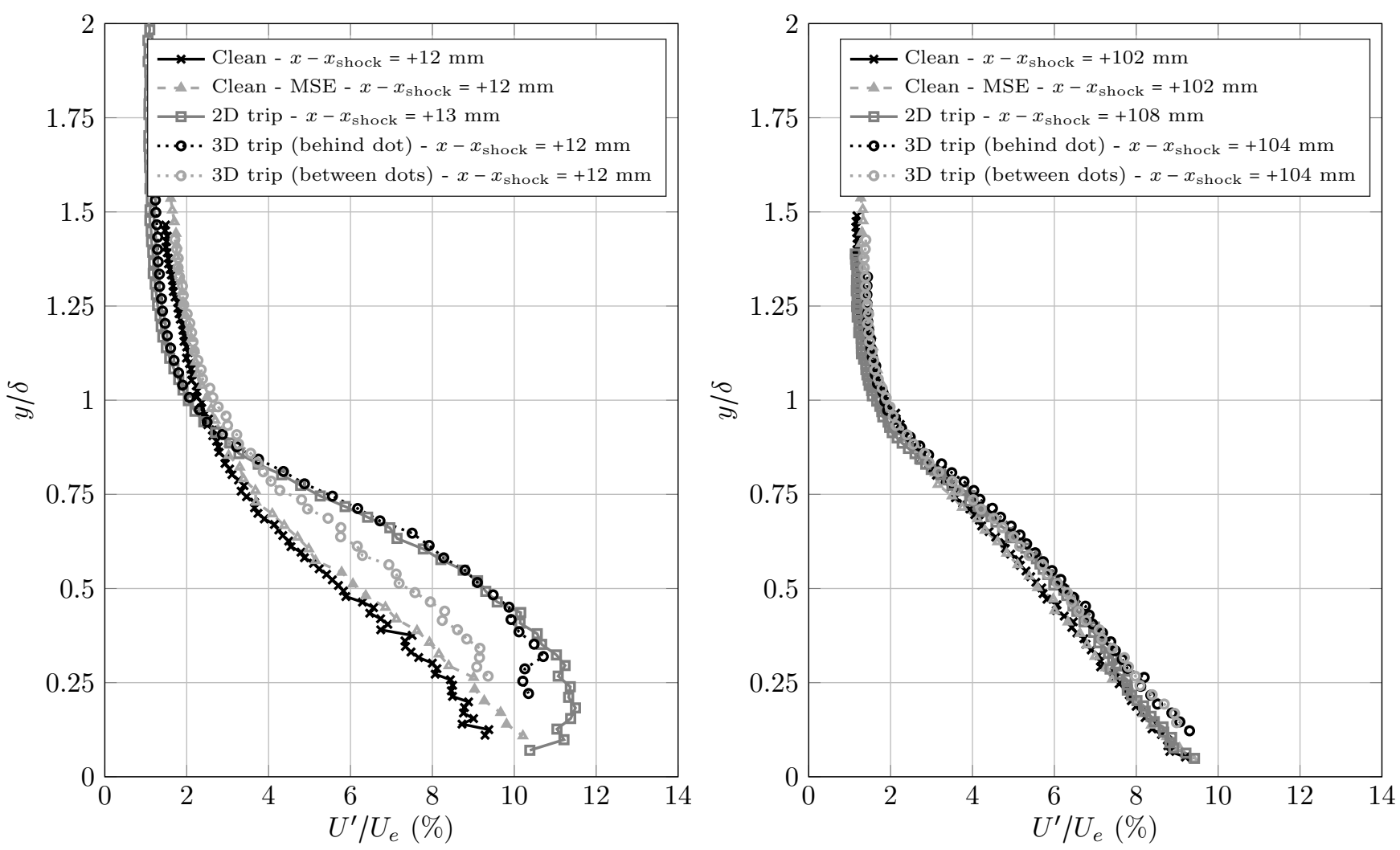

Fig. 15 Variation of fluctuation intensity through boundary layers downstream of the interaction: a) $x-x_{\text {shock }} \sim+12 \mathrm{~mm}$; b) $x-x_{\text {shock }} \sim+100 \mathrm{~mm}$. 
Similar behaviours are evidenced in the fluctuation intensity profiles of Fig. 15. Shortly downstream of the shock, the clean cases already show an equilibrium turbulent boundary layer fluctuation intensity distribution. Behind the trips the impact of the shock is clear, as the turbulence level is increased and the peak intensity is displaced from the wall. By $100 \mathrm{~mm}$ downstream, all cases have recovered to a typical turbulent distribution.

A curiosity observed in Fig. 15a is a difference in the fluctuation levels just outside the boundary layers. The intensity is marginally higher in the two clean cases and between the trip dots $(2.0-2.3 \%)$ than with trips (1.7\%). Further along the plate, in Fig. 15b, this distinction has disappeared. In order to examine how this variation develops, the intensity level from each boundary layer traverse for each configuration was extracted at $y=1.1 \delta$ and plotted in Fig. 16. It can be seen that while the unsteadiness is immediately greater near the shock for the clean cases, it rapidly dies away: by $x-x_{\text {shock }} \sim 30 \mathrm{~mm}$, the values are $1.7 \%$ for the laminar interactions, $1.5 \%$ with trips. Additionally, the short-lived increase in fluctuation level is confined to near the boundary layer edge: high speed schlieren imaging revealed no notable unsteadiness for any interaction.

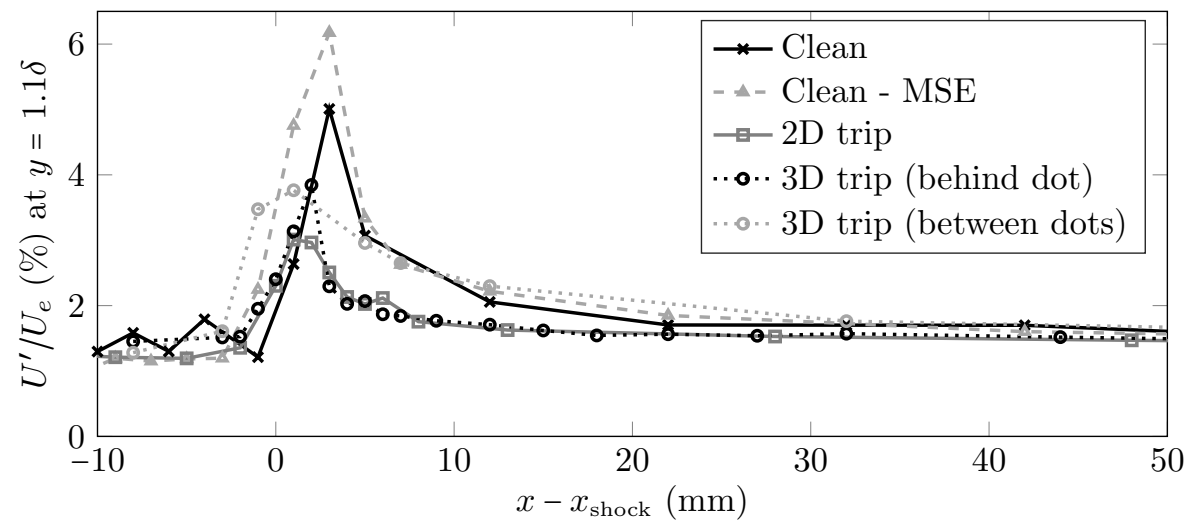

Fig. 16 Fluctuation intensity just above the boundary layer for each of the interactions.

The increased fluctuation level may be connected to the unsteadiness at the shock foot (coinciding with reattachment) seen by Swoboda \& Nitsche (1996) on a laminar aerofoil. Careful examination of their reported intensity contours also show something similar to that observed here, in the region near the wall just downstream of the rear leg. [14] A possible source could be the occurrence of 'rollers' observed at the edge of laminar separation bubbles: span-wise coherent vortices that apparently form as a result of 'roll up' of Kelvin-Helmholtz instabilities developing in the shear layer. These have been much observed in low speed experiments. [e.g. 43-45] Kurelek et al. (2016) determined that they break down soon after reattachment and occasionally migrate outside the boundary layer which could explain the extent of the presently observed phenomenon. [45] They have also been observed at high speed, computationally at least, for oblique shock-induced laminar separation bubbles. [46] 


\section{Discussion}

As laminar boundary layers separate so easily, a large, detrimental separation was feared in the clean cases. However, it is clear from the schlieren images and boundary layer measurements that while the observed separation bubble may be very long, especially in terms of $\delta_{1}$, it never becomes very thick. With hindsight, this could have been predicted from oblique shock relations [47] (or the method of characteristics, following Messiter et al.'s supposition that the front leg compression does not coalesce into a shock [13]): as the pressure rise through the front leg is so small $(<6 \%)$, the amount of flow turning associated with it is also small $\left(<1^{\circ}\right)$. This physically corresponds to the 'wedge angle' of the separation bubble, and so the separation grows slowly and remains shallow (height much less than its length). Therefore the separation bubble has very little displacement effect on the outer flow, and the feared extreme flow deviation does not occur. Further, high frame rate imaging showed that the shock structure was very steady.

LDV measurements downstream of the clean interaction confirm that the boundary layer has transitioned (as expected, because $R e_{x_{\text {shock }}}$ is close to the natural transition Reynolds number - see Fig. 10 - and the separation will make the boundary layer less stable) and the impact of the separation is minimal. In fact, compared to the tripped cases, the flow rehabilitates downstream of the laminar interaction far more quickly, presumably accelerated by transition and reattachment. Despite having the same shock strength, the turbulent interactions lead to an extended region downstream of the shock with low momentum flow near the wall, along with increased turbulence intensity, which could negatively affect the wake if the interaction were occurring close to the trailing edge of an aerofoil or compressor blade.

A point to note is that the interactions each have different incoming boundary layer thicknesses, which may hide scale effects. Indeed, Fig. 13a shows that $\delta$ increases by a factor of around five through the clean interactions, due to the combination of separation, transition, the lambda shock and reattachment. Nonetheless, as laminar boundary layers are typically thinner than their tripped or turbulent counterparts, the resulting boundary layer thickness behind the interaction can still be smaller, as observed here. This may indicate that a sufficiently discreet trip which avoids a significant increase in $\delta$ could result in a thinner downstream boundary layer than in the clean cases. However, it is difficult to imagine a more subtle trip than a layer of paint thinner than a human hair - which still had a greater impact on the thickness than did the laminar interaction - and there would still be the slower post-shock recovery of the turbulent interaction.

One qualification to make is that these experiments have been undertaken at Reynolds numbers close to natural transition, and so, when provoked by the interaction, transition is able to occur shortly after separation. If $R e_{x_{\text {shock }}}$ were lower, then the situation could be different - perhaps the separation bubble or even 
laminar flow persisting downstream of the interaction - but as many practical applications are likely to be in a similar Reynolds number range, the observed behaviour is pertinent.

\section{Conclusions}

This paper has presented normal shock wave-boundary layer interactions for laminar and tripped boundary layers on a flat plate at a Mach number of 1.2 , to investigate how the incoming boundary layer state affects the development of the flow downstream of the shock. It was anticipated that the clean interactions would be detrimental to the global flow because of the laminar boundary layer's proclivity to separate - separation was expected to impact the downstream flow development by greatly increasing the boundary layer thickness, potentially introducing large-scale unsteadiness and generally leading to an overall increase in drag; despite laminarity being favoured upstream of the shock for its lower skin friction.

However, this has not been the case. The interaction with the laminar incoming boundary layer did cause separation and a resulting lambda structure, but with no increase in $\delta$ compared to the tripped interactions. Indeed, a single layer of paint caused a greater increase in downstream boundary layer thickness than the laminar interaction. In terms of mean and fluctuating profiles, shape factor and skin friction coefficient, the boundary layer far downstream of the interaction showed little dependence on whether or not the incoming boundary layer had been tripped before the shock. In the near downstream region, the turbulent interaction was actually more detrimental than the laminar interaction, with the boundary layers requiring a long rehabilitation to an equilibrium turbulent profile while exhibiting increased fluctuations.

The lack of impact seen due to the laminar interaction is actually attributed to the fact that laminar boundary layers do separate so easily: the low pressure rise at separation corresponds to only a weak compression through the oblique front leg, which necessarily only turns the flow slightly, and so the separation bubble formed is very thin. The flow lofted over this bubble is then presumed to transition before the larger pressure rise of the rear leg, and the rest of the interaction is benign, with reattachment and a rapid rehabilitation to an equilibrium turbulent profile.

The present interaction strength of Mach 1.2 was chosen as a value likely to separate a laminar boundary layer, but not a turbulent one. An interesting extension to the current work would be to investigate the impact of instigating transition ahead of interactions at higher Mach numbers, such as 1.3 or 1.4, where even the tripped or naturally turbulent boundary layer would be expected to separate. A comparison between the separation sizes and boundary layer development downstream of reattachment could provide further valuable physical insight into normal shock wave-transitional boundary layer interactions. 


\section{Acknowledgements}

The authors would like to acknowledge the TFAST Project of the European Commission's 7th Framework Programme for funding the work. They also wish to thank Dave Martin, Sam Flint, Tony Luckett, John Hazlewood and Ben Stock for operating and maintaining the CUED No. 1 Supersonic Tunnel, and Kevin Bullman for manufacturing the flat plates. The UK's National Wind Tunnel Facility initiative additionally supports the CUED No. 1 \& 2 Supersonic Tunnels.

\section{References}

[1] Ackeret, J., Feldman, F., and Rott, N., "Investigations of Compression Shocks and Boundary Layers in Gases Moving at High Speed", NACA Technical Memorandum, 1113, 1947.

[2] Délery, J. M., "Shock wave/turbulent boundary layer interaction and its control", Progress in Aerospace Sciences, 22:209-280, 1985, doi:10.1016/0376-0421(85)90001-6.

[3] Babinsky, H. and Harvey, J. K., Shock Wave-Boundary-Layer Interactions, Cambridge University Press, 2011, doi:10.1017/CBO9780511842757.

[4] Gadd, G. E., "Interactions between Normal Shock Waves and Turbulent Boundary Layers", Aeronautical Research Council Reports 83 Memoranda, 3262, 1961.

[5] Sawyer, W. G. and Long, C. J., "A Study of Normal Shock-Wave Turbulent Boundary-Layer Interactions at Mach Numbers of 1.3, 1.4 and 1.5", NASA Technical Report, 82099, 1982.

[6] Délery, J. M. and Marvin, J. G., "Shock-wave boundary layer interactions", AGARDograph, 280, 1986.

[7] Atkin, C. J. and Squire, L. C., "A study of the interaction of a normal shock wave with a turbulent boundary layer at Mach numbers between 1.30 and 1.55", European Journal of Mechanics B (Fluids), $11,1992$.

[8] Davidson, T. S. C. and Babinsky, H., "An investigation of interactions between normal shocks and transitional boundary layers", 44th AIAA Fluid Dynamics Conference, 3334, 2014, doi:10.2514/6.20143334 .

[9] Ogawa, H. and Babinsky, H., "Wind-Tunnel Setup for Investigations of Normal Shock Wave/Boundary Layer Interaction Control", AIAA Journal, 44(11):2803-2805, 2006, doi:10.2514/1.24370.

[10] East, L. F., "The application of a laser anemometer to the investigation of shock-wave boundary-layer interactions", AGARD Applications of Non-Intrusive Instruments in Fluid Flow Research, 10, 1976.

[11] Liepmann, H. W., "The Interaction Between Boundary Layer and Shock Waves in Transonic Flow", Journal of the Aeronautical Sciences, 13(12):623-637, 1946, doi:10.2514/8.11473.

[12] Seddon, J., "The Flow Produced by Interaction of a Turbulent Boundary Layer with a Normal Shock Wave of Strength Sufficient to Cause Separation", Aeronautical Research Council Reports \& Memoranda, $3502,1960$.

[13] Messiter, A. F., Feo, A., and Melnik, R. E., "Shock-Wave Strength for Separation of a Laminar Boundary Layer at Transonic Speeds", AIAA Journal, 9(6):1197-1198, 1971.

[14] Swoboda, M. and Nitsche, W., "Shock boundary-layer interaction on transonic airfoils for laminar and turbulent flow", Journal of Aircraft, 33(1):100-108, 1996, doi:10.2514/3.46909.

[15] Arnal, D. and Délery, J. M., "Laminar-turbulent transition and shock wave/boundary layer interaction", In RTO AVT Lecture Series on Critical Technologies for Hypersonic Vehicle Development, 2004. 
[16] Alam, M. and Sandham, N. D., "Direct numerical simulation of short' laminar separation bubbles with turbulent reattachment", Journal of Fluid Mechanics, 410:1-28, 2000, doi:10.1017/S0022112099008976.

[17] Drela, M., "Transonic low-Reynolds number airfoils", Journal of Aircraft, 29(6):1106-1113, 1992, doi:10.2514/3.46292.

[18] Tain, L. and Cumpsty, N. A., "Compressor blade leading edges in subsonic compressible flow", Proceedings of the Institution of Mechanical Engineers, Part C: Journal of Mechanical Engineering Science, 214(1):221-242, 2000.

[19] Smits, A. J., Hayakawa, K., and Muck, K.-C., "Constant Temperature Hot-wire Anemometer Practice in Supersonic Flows - Part I: The Normal Wire", Experiments in Fluids, 1:83-92, 1983, doi:10.1007/BF00266260.

[20] Smits, A. J. and Dussauge, J.-P. P., "A survey of measurements and measuring techniques in rapidly distorted compressible turbulent boundary layers: Hot-wire anemometry in supersonic flow", AGARDograph, 315(5), 1988.

[21] Horstman, C. C. and Rose, W. C., "Hot-wire anemometry in transonic flow", AIAA Journal, 15(3): 395-401, 1977, doi:10.2514/3.7331.

[22] Rong, B. S., Tan, D. K., and Smits, A. J., "The calibration of the constant temperature normal hotwire anemometer in transonic flow", University of Princeton, Department of Mechanical 83 Aerospace Engineering Report, MAE-1696, 1985.

[23] Zhong, S., An interferometric study of organized structures in compressible turbulent flows, $\mathrm{PhD}$ thesis, University of Cambridge, 1993.

[24] Hinchliffe, R. A., The interaction of a normal shock with a non-equilibrium boundary layer, PhD thesis, University of Cambridge, 1993.

[25] Schrader, L.-U., Brandt, L., Mavriplis, C., and Henningson, D. S., "Receptivity to free-stream vorticity of flow past a flat plate with elliptic leading edge", Journal of Fluid Mechanics, 653:245-271, 2010, doi:10.1017/S0022112010000376.

[26] Lin, N., Reed, H. L., and Saric, W. S., "Effect of leading-edge geometry on boundary-layer receptivity to freestream sound", Instability, Transition, and Turbulence, pages 421-440, 1992, doi:10.1007/978-1$4612-2956-8 \_42$.

[27] van Driest, E. R. and Blumer, C. B., "Boundary layer transition at supersonic speeds - three dimensional roughness effects (spheres)", AFOSR Scientific Report, AFOSR 1493, 1961.

[28] Bernardini, M., Pirozzoli, S., Orlandi, P., and Lele, S. K., "Parameterization of Boundary-Layer Transition Induced by Isolated Roughness Elements", AIAA Journal, 52(10):2261-2269, 2014, doi:10.2514/1.J052842.

[29] Settles, G. S., Schlieren and Shadowgraph Techniques: Visualizing Phenomena in Transparent Media, Springer-Verlag Berlin Heidelberg, 2001, doi:10.1007/978-3-642-56640-0.

[30] Colliss, S. P., Vortical structures on three-dimensional shock control bumps, PhD thesis, University of Cambridge, 2014.

[31] Lai, W., Shakal, J., and Troolin, D., "Accuracy, Resolution, and Repeatability of Powersight PDPA and LDV Systems", TSI Technical Note, P/N 500152, 2013.

[32] McLaughlin, D. K. and Tiederman, W. G., "Biasing correction for individual realization of laser anemometer measurements in turbulent flows", Physics of Fluids, 16(1973):2082-2088, 1973, doi:10.1063/1.1694269. 
[33] Sun, C.-C. and Childs, M. E., "A modified wall wake velocity profile for turbulent compressible boundary layers.", Journal of Aircraft, 10(6):381-383, 1973, doi:10.2514/3.44376.

[34] van Driest, E. R., "Turbulent Boundary Layer in Compressible Fluids", Journal of the Aeronautical Sciences, 18(3):145-160, 1951, doi:10.2514/2.7048.

[35] Coles, D., "The law of the wake in the turbulent boundary layer", Journal of Fluid Mechanics, 1(02): 191, 1956, doi:10.1017/S0022112056000135.

[36] Musker, A. J., "Explicit Expression for the Smooth Wall Velocity Distribution in a Turbulent Boundary Layer", AIAA Journal, 17(6):655-657, 1979, doi:10.2514/3.61193.

[37] Titchener, N. A., Colliss, S. P., and Babinsky, H., "On the calculation of boundary-layer parameters from discrete data", Experiments in Fluids, 56(8):159, 2015, doi:10.1007/s00348-015-2024-5.

[38] Kooi, J. W., "Experiment on transonic shock wave boundary layer interaction", AGARD Flow Separation, 10(30), 1975.

[39] Pearcey, H. H., "The Indication of Boundary-Layer Transition On Aerofoils in the N.P.L. 20 in. x 8 in. High Speed Wind Tunnel", Aeronautical Research Council Current Papers, 10, 1948.

[40] Chapman, D. R., Kuehn, D. M., and Larson, H. K., "Investigation of Separated Flows in Supersonic and Subsonic Streams with Emphasis on the Effect of Transition", NACA Report, 1356, 1958.

[41] Davidson, T. S. C. and Babinsky, H., "Transition location effects on normal shock wave-boundary layer interactions", 53rd AIAA Aerospace Sciences Meeting, 1975, 2015, doi:10.2514/6.2015-1975.

[42] Davidson, T. S. C. and Babinsky, H., "Influence of transition on the flow downstream of normal shock wave-boundary layer interactions", 54th AIAA Aerospace Sciences Meeting, 0044, 2016, doi:10.2514/6.2016-0044.

[43] Serna, J. and Lázaro, B. J., "Experiments on Natural Transition in Separation Bubbles", Procedia IUTAM, 2210-9838:496-502, 2015, doi:10.1016/j.piutam.2015.03.078.

[44] Serna, J. and Lázaro, B. J., "On the laminar region and the initial stages of transition in transitional separation bubbles", European Journal of Mechanics B: Fluids, 49:171-183, 2015, doi:10.1016/j.euromechflu.2014.08.006.

[45] Kurelek, J. W., Lambert, A. R., and Yarusevych, S., "Coherent Structures in the Transition Process of a Laminar Separation Bubble", AIAA Journal, 54(8):1-15, 2016, doi:10.2514/1.J054820.

[46] Sivasubramanian, J. and Fasel, H. F., "Numerical Investigation of Shock-Induced Laminar Separation Bubble in a Mach 2 Boundary Layer", 45th AIAA Fluid Dynamics Conference, 2641, 2015, doi:10.2514/6.2015-2641.

[47] Shapiro, A. H., The Dynamics and Thermodynamics of Compressible Fluid Flow, The Ronald Press Company, New York, 1954. 